\title{
Medio siglo de evolución del concepto de Relaciones Públicas (1970-2020). Artículo de revisión
}

\author{
Half a century in the evolution of the \\ Concept of public relations (1970-2020). Review article
}

\author{
María-Teresa García-Nieto; Mónica Viñarás-Abad; Francisco Cabezuelo-Lorenzo
}

Cómo citar este artículo:

García-Nieto, María-Teresa; Viñarás-Abad, Mónica; Cabezuelo-Lorenzo, Francisco (2020). “Medio siglo de evolución del concepto de Relaciones Públicas (1970-2020). Artículo de revisión". El profesional de la información, v. 29, n. 3, e290319.

https://doi.org/10.3145/epi.2020.may.19

Artículo recibido el 08-11-2019 Aceptación definitiva: 12-03-2020

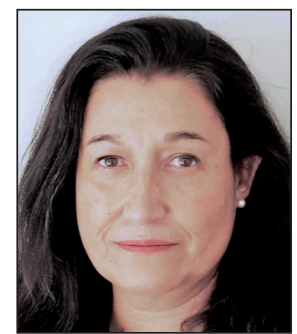

María-Teresa García-Nieto https://orcid.org/0000-0002-6235-511X

Universidad Complutense de Madrid Facultad de Ciencias de la Información Avenida Complutense, 3. 28040 Madrid, España xyz@ccinf.ucm.es

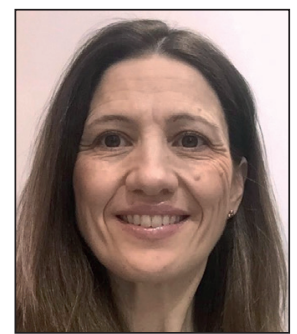

Mónica Viñarás-Abad https://orcid.org/0000-0001-8792-5927

Universidad San Pablo CEU

Facultad de Humanidades y Ciencias de la Comunicación

Paseo de Juan XXIII, 8. 28040 Madrid, España monica.vinarasabad@ceu.es

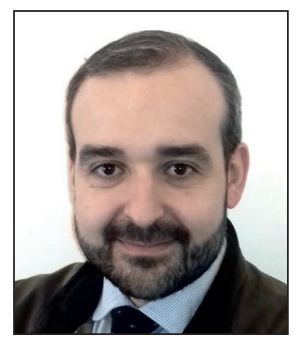

\author{
Francisco Cabezuelo-Lorenzo $\triangle$ \\ https://orcid.org/0000-0002-9380-3552 \\ Universidad Complutense de Madrid \\ Facultad de Ciencias de la Información \\ Avenida Complutense, 3. \\ 28040 Madrid, España \\ fcabezuelo@ucm.es
}

\section{Resumen}

Este artículo de revisión presenta una exhaustiva reflexión epistemológica sobre la evolución del concepto de Relaciones Públicas durante el último medio siglo (1970-2020). Se han recogido de manera exploratoria las definiciones más notorias referidas en los materiales académicos, manuales, monográficos y artículos científicos sobre Relaciones Públicas. Nuestro objetivo es comprobar cómo ha evolucionado el concepto de Relaciones Públicas y cómo se ha ido modelando con las sucesivas definiciones enunciadas por expertos, académicos e investigadores. Se evidencia como durante los años setenta del siglo XX, pese al claro disenso sobre la concepción de las Relaciones Públicas (que discurre desde el esfuerzo organizado hasta el arte o la ciencia social) existe ya un común reconocimiento de su naturaleza persuasiva y de su fundamentación en las ciencias sociales. En la década de los años ochenta, las Relaciones Públicas, influenciadas por las teorías de la gestión empresarial, se identifican como una función gerencial y directiva de la comunicación. Y, a partir de los años noventa, las Relaciones Públicas socialmente responsables avanzan hacia unos objetivos de mutuo entendimiento con los públicos. Las últimas definiciones que reafirman el carácter gerencial coexisten con otras que las conciben como un proceso estratégico de comunicación. Este estudio confirma su naturaleza gerencial, sus objetivos de mutuo beneficio y recíproco entendimiento entre la organización y sus públicos, y su carácter socialmente responsable. Se consolida por tanto una conceptualización alejada de los objetivos comerciales y económicos del marketing, con los que a veces erróneamente se confunde. Finalmente, este trabajo señala en sus conclusiones los elementos definidores de las actuales Relaciones Públicas. 


\title{
Palabras clave
}

Relaciones Públicas; Historia de las Relaciones Públicas; Epistemología de las Relaciones Públicas; Relaciones Públicas gerenciales; Relaciones Públicas estratégicas; Relaciones Públicas persuasivas; Gestión de la comunicación; Responsabilidad social corporativa; Marketing; Artículo de revisión.

\begin{abstract}
This review article offers an exhaustive epistemological work on the evolution of the concept of public relations during the last half century (1970-2020). For this purpose, the most referred definitions from the academy, manuals, books and scientific articles focused on public relations have been explored and summarized. The objective is to verify how the concept of public relations has evolved and how it has been modeled with the successive definitions set forth by experts, academics and researchers. Thus, as in the decade of the seventies of the 20th century, despite the clear dissent on the conception of public relations, which goes from organized effort to art or social science, there is already a common recognition of its nature persuasive and its foundation in the social sciences. In the 1980s, public relations, influenced by business management, are identified as a managerial and directive function of communication. And, starting in the 1990s, socially responsible public relations are moving towards goals of mutual understanding with the public. The latest definitions that reaffirm the managerial nature, coexist with others that conceive them as a strategic communication process. The study confirms its managerial nature, in its objectives of mutual benefit and mutual understanding between the organization and its public, along with its socially responsible nature. It is therefore finally a concept away from the commercial and economic objectives of marketing, with which it is sometimes mistakenly confused. Finally, in the conclusions, this article considers the defining elements of current public relations.
\end{abstract}

\section{Keywords}

Public Relations; History of Public Relations; PR Epistemology; Managerial PR; Strategic Public Relations; Persuasive Public Relations; Communication Management; Corporate Social Responsibility; Marketing; Review article.

\section{Introducción}

En las últimas dos décadas, la sociedad de la información y la comunicación, devenida en sociedad del conocimiento, ha derribado las tradicionales fronteras entre las profesiones comunicativas, organizacionales y empresariales, produciendo una gran confusión terminológica. La comunicación tradicional bidireccional se ha visto influida por los nuevos roles de los profesionales digitales, como los community managers (Mañas-Viniegra; Jiménez-Gómez, 2019), que afectan también a los espacios de reflexión académica y conceptual o de formación en el campo de Relaciones Públicas (Matilla; Cuenca-Fontbona; Compte-Pujol, 2017).

Los conceptos precedentes sustentadores de teorías y proposiciones sobre la gestión comunicativa y empresarial se han quedado en algunos casos obsoletos. Los términos de Relaciones Públicas, Publicidad, Periodismo, Comunicación Corporativa, Marketing y otros, se intercalan y solapan en una increíble rueda de definiciones ante el reto digital transmedia y crossmedia (Hellín-Ortuño; Trindade; García-López, 2019), la comunicación organizacional digital (García-Orosa, 2019) y los nuevos contextos comunicacionales internacionales (Manfredi-Sánchez, 2020).

Por ello se hace necesario definir y reafirmar el marco conceptual de las Relaciones Públicas, desde sus precedentes científicos hasta el momento presente, con el fin de dejar sentados sus conceptos básicos y nucleares, con el objetivo de ampliar y potenciar los nuevos modelos de investigación, docencia y práctica profesional. El concepto de Relaciones Públicas se ha ido modelando desde que el norteamericano Bernays (1990) dictara las primeras lecciones sobre este tema en el ámbito universitario en 1923, y Harlow (1976) creara en 1939 el primer plan de estudios de esta especialidad en la Stanford University. Sin embargo, las reflexiones más profundas no se hicieron en sus inicios, sino en los últimos cincuenta años.

El objetivo de este trabajo es comprobar cómo se ha ido forjando el concepto actual de Relaciones Públicas durante el último medio siglo. De este modo, se pretende ofrecer una revisión y actualización epistemológica de este concepto a partir de las definiciones más notorias enunciadas por expertos e investigadores, frecuentemente referidas en los textos académicos, manuales, monografías, documentos institucionales y de trabajo, y en artículos científicos sobre esta materia.

\section{Dimensión persuasiva de las Relaciones Públicas}

En los años sesenta del pasado siglo las actividades de Relaciones Públicas estaban fundamentadas en el tradicional principio conceptual simple y concreto de "hacerlo bien y hacerlo saber". Los profesionales definían su trabajo como un proceso transparente basado en informaciones verídicas y confirmadas sobre las actuaciones de personas e instituciones. Las Relaciones Públicas no debían ser en ningún caso una mera tapadera de prácticas incorrectas y socialmente reprobables, como era costumbre en los ancestrales programas y campañas de los tiempos remotos, donde se trataba de encubrir informaciones y acontecimientos. Por ello era obligado trabajar bien, en cuanto al quehacer profesional, y comunicarlo bien con un intercambio de mensajes correcto y adecuado a los objetivos propuestos. Este principio atribui- 
do de forma exclusiva a las Relaciones Públicas, en un inútil reduccionismo, es parte importante de toda actividad comunicativa (salvo en los obligados casos del necesario secreto), sea ésta informativa, interpretativa, opinativa, educativa, de entretenimiento, o persuasiva. A partir de este sencillo planteamiento, numerosos investigadores y profesionales de las Relaciones Públicas realizaron un gran esfuerzo conceptualizador para definir epistemológicamente la realidad teórica, técnica y práctica de su trabajo. Una primera definición, convertida ya en un clásico, fue la de Cutlip y Center que definieron las Relaciones Públicas como

"el esfuerzo planteado para influir en la opinión por medio de una actuación aceptable y de la comunicación recíproca" (Cutlip; Center, 1978).

Otra célebre definición del mismo período es la de Carlson, que afirmaba que

"las Relaciones Públicas constituyen un esfuerzo organizado para comunicar información y modificar las actitudes y el comportamiento en beneficio de un cliente o una causa" (Carlson, 1979).

Ambas definiciones hablan de las Relaciones Públicas como un esfuerzo organizado y planificado para transmitir informaciones, en un proceso de comunicación recíproca que implica un intercambio de mensajes, con el deseo de modificar actitudes y comportamientos en los públicos receptores. La nueva conceptualización, muy sencilla y adecuada, suponía una buena manera de iniciar un debate sobre las formas y los modos propios de las Relaciones Públicas.

El 12 de agosto de 1978 se celebró en Ciudad de México la primera Asamblea Mundial de Asociaciones de Relaciones Públicas con la asistencia de sus presidentes, los más importantes y prestigiosos investigadores y profesionales del sector. Preocupados por determinar el marco definidor de las Relaciones Públicas, redactaron la Declaración de México donde articularon claramente las líneas de fuerza de su trabajo diario. Acordaron que el ejercicio de las Relaciones Públicas se fundamenta en el arte y la ciencia social de analizar tendencias, predecir consecuencias, asesorar a los líderes de las organizaciones y poner en práctica programas planificados de acción que servirán a los intereses de la organización y del público. Por primera vez aparecieron nuevos términos posibilitadores de una mejor delimitación conceptual como arte, ciencia social, análisis de tendencias, práctica de asesoría, o programación planificada, con el objetivo concreto de realizar un trabajo útil y adecuado a los intereses de los distintos públicos. El camino a seguir por la investigación en Relaciones Públicas estaba trazado.

En 1980, la Public Relations Society of America (PRSA) proporcionó a los profesionales de esta nueva actividad dos nuevos asertos dignos de resaltar:

- el primero expresaba claramente que "las Relaciones Públicas ayudan a una organización y a sus públicos a adaptarse mutuamente";

- el segundo matizaba y delineaba ideas posteriores. Estimaba que "son un esfuerzo de una organización para ganar la cooperación de grupos de gente" (Lesly, 1981, p. 32).

Los nuevos términos incorporados a las Relaciones Públicas radicaban en el concepto de adaptación mutua y el de cooperación con las gentes.

En la bibliografía europea y española sobre estos temas, todavía en años posteriores podemos encontrar algunas definiciones de esta misma naturaleza, como la ofrecida por Westphalen y Piñuel-Raigada, quienes definieron las Relaciones Públicas como el

"conjunto de medios y técnicas utilizadas por una organización, pública o privada, para comunicarse con sus diferentes públicos (socios, personal, accionistas, consumidores, proveedores, comunidades locales, poderes públicos, administración), con el objetivo de acrecentar su notoriedad y de mejorar su imagen" (Westphalen; Piñuel-Raigada, (1993).

Además, añadían que

"las publicaciones, las operaciones de mecenazgo y patrocinio, la organización de conferencias y manifestaciones, la participación en salones y ferias profesionales, el montaje de jornadas de puertas abiertas y otros, constituyen otros tantos útiles de las Relaciones Públicas" (Westphalen; Piñuel-Raigada, 1993, p. 1127-1128).

Independientemente del lenguaje utilizado, muy de los años finales del pasado siglo, esta forma de entender las Relaciones Públicas tiene la virtud de proporcionar al estudioso y al profesional un elenco de técnicas y herramientas necesarias para su trabajo.

Al analizar estas definiciones se aprecia un claro desacuerdo entre los autores a la hora de identificar la naturaleza de las Relaciones Públicas. Estas discrepancias nos llevan a reformular varias cuestiones: ¿Qué son en definitiva las Relaciones Públicas? ¿Un esfuerzo organizado para comunicar o para influir? ¿Son sólo una pieza en la cadena de negocio o también tienen algo de arte? ¿Son una ciencia social o un conjunto de técnicas? ¿No son acaso todo ello al mismo tiempo? En este debate se observa un cierto lazo de unión sobre la existencia de las Relaciones Públicas y su gran utilidad en las sociedades avanzadas. Pero sobre todo, y

Las Relaciones Públicas deben estar dotadas de un extraordinario carácter persuasivo 
esto es importante, estas afirmaciones parecen remitir, de una forma más o menos explícita, a entender las Relaciones Públicas como una tarea persuasiva. Deben estar dotadas de un extraordinario carácter persuasivo.

En este sentido, Bernays afirmaba en el muy lejano año 1952 que el término Relaciones Públicas tenía

"tres significados": (1) la información que se da al público; (2) persuasión dirigida al público para modificar sus actitudes y conductas; (3) esfuerzo para integrar las actitudes y acciones de una institución con las de sus públicos y la de sus públicos con las de esa institución" (Bernays, 1970, p. 3).

Este matiz lo reforzó Bettinghaus cuando afirmó que para poder atribuir el carácter persuasivo a cualquier proceso comunicativo debe existir

"el intento consciente por parte del individuo de cambiar las actitudes, creencias o conductas de otro individuo o grupos de individuos mediante la transmisión de algún mensaje" (Bettinghaus, 1987, p. 3-4).

Por todo ello, y en consonancia con los razonamientos anteriores, es posible afirmar que las Relaciones Públicas utilizan fundamentalmente las formas y los modos de la comunicación persuasiva, independientemente de cómo se definan, tanto si son un esfuerzo organizado, una clase de negocio, unas simples técnicas, un arte o una ciencia.

A partir de este momento, el estatuto epistemológico de las Relaciones Públicas incorporó el primero de sus conceptos fundamentales. Podemos hablar sin lugar a equivocarnos, de la indudable dimensión persuasiva de las Relaciones Públicas, de la efectiva vinculación entre éstas y los procesos persuasivos, con su bagaje científico, postulados vigentes en nuestros días. Esta naturaleza persuasiva constituía para Noguero-Grau (1995) el paradigma único y exclusivo para la investigación y la docencia universitaria de las Relaciones Públicas. Se construyó así una nueva concepción de las Relaciones Públicas, de carácter multidisciplinar, con el aporte epistemológico de la psicología social aplicada, en los términos del cambio de actitudes y sus derivados motivacionales-percepciones, creencias, imágenes, predisposiciones, opiniones, conductas- y de las modernas teorías de la comunicación, en especial de la llamada comunicación colectiva.

Enmarcar las Relaciones Públicas en una nítida condición persuasiva ha significado para ellas la consolidación de su carácter científico, más allá del simple enunciado de sus conceptos, tanto en el orden de alcanzar ciertos objetivos como en el diseño de las estrategias más convenientes para conseguirlos. La dimensión persuasiva de las Relaciones Públicas ofreció a los profesionales la posibilidad, hasta entonces falaz, de avalar la eficacia de sus acciones, programas y campañas, mediante la planificación estratégica, dejando atrás el viejo modo de trabajar basado únicamente en la intuición y la experiencia. La fundamentación epistemológica de las Relaciones Públicas, caracterizada por una fértil aportación multidisciplinar, representó un relevante aldabonazo entre los profesionales y confirmó definitivamente la madurez teórica, técnica y práctica de esta disciplina.

\section{Dimensión directiva de las Relaciones Públicas}

Alrededor de 1980 los teóricos más innovadores dieron un paso adelante en la elaboración del marco conceptual de estos conocimientos al ofrecer creativas respuestas a los interrogantes planteados en décadas precedentes. El estatuto epistemológico de las Relaciones Públicas se enriqueció con un innovador concepto. Las Relaciones Públicas son una filosofía, una política, gerencial o directiva. ¿Cuáles son los motivos para recurrir a los conceptos gerenciales en el momento de definir la naturaleza de las Relaciones Públicas? La razón se encuentra en una de las aportaciones científicas que, a partir de entonces, han empapado y empapan el pensar y el hacer de las Relaciones Públicas. Son los presupuestos del llamado business management o gerencia de negocios, una nueva cultura que había irrumpido ya con fuerza en el mundo empresarial cambiando las formas de gestión de los tiempos.

Los pioneros en esos cambios metodológicos fueron Moore y Canfield al afirmar que el carácter de las Relaciones Públicas se fundamenta en

"una filosofía social de la gerencia que deliberadamente y con resplandeciente egoísmo sitúa en primer plano los diversos intereses del público en cada decisión del negocio"

para concluir con firmeza:

"Las Relaciones Públicas son una filosofía social expresada en decisiones políticas" (Moore; Canfield, 1980, pp. 22-23).

En este mismo orden de cosas, Marston aceptó sin reservas la definición que recogió de Griswold en la que estimaba que

“las Relaciones Públicas son la función gerencial que evalúa las actitudes públicas, identifica las políticas y los procedimientos de una organización con el interés público, y ejecuta un programa de acción y comunicación para ganar aceptación y entendimientos públicos" (Marston, 1979, p. 6).

Por su parte, años más tarde, conjuntamente Grunig y Hunt $(1984$, p. 6) y por separado (Grunig, 1992, p. 4) incidieron en este aspecto con una síntesis clarificadora al anunciar que las Relaciones Públicas son

"la gerencia de la comunicación entre una organización y sus públicos".

Por su parte, Center y Jackson afirmaron rotundamente que

“las Relaciones Públicas son responsabilidad de la dirección” (Center; Jackson, 1995, p. 1). 


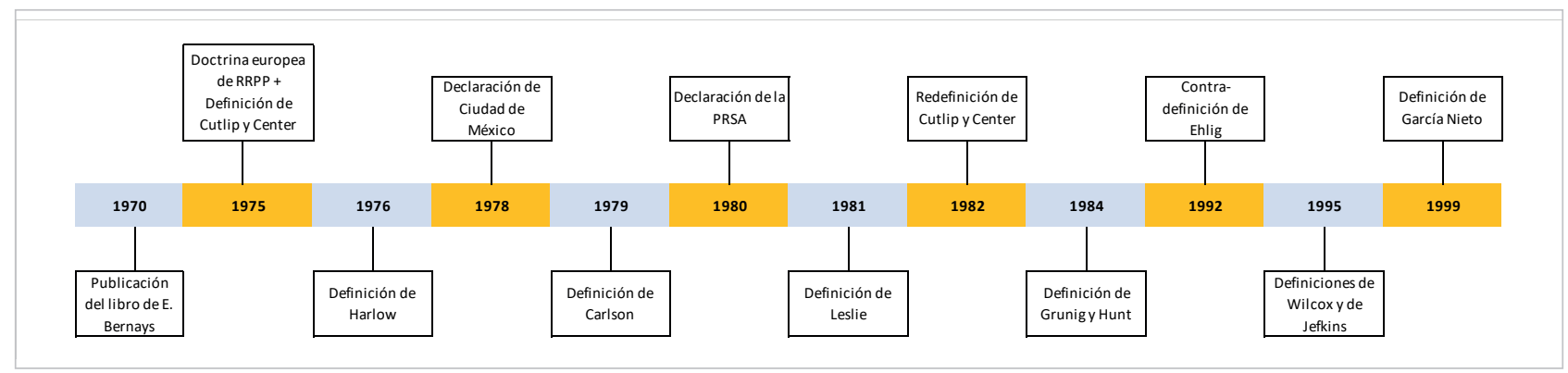

Cronograma 1. Conceptualización de las RRPP de 1970 a 1999

Todos concebían las Relaciones Públicas como una forma de entender la gestión, la gerencia o la dirección de una organización o persona, en lo relativo a su relación con los diferentes públicos, con el fin de garantizar la formación y el mantenimiento de una determinada imagen pública, así como una actitud favorable de sus públicos hacia dicha organización o persona. Pese a la indudable dimensión directiva o gerencial de las Relaciones Públicas se confirmaba también el carácter persuasivo de estas tareas en lo referente a sus objetivos y a la necesidad de aplicar la investigación científica adecuada.

La idea de las Relaciones Públicas de esta época, que resume e integra las diferentes aportaciones sobre su innegable dimensión gerencial, se concreta de la manera siguiente: las Relaciones Públicas se configuran como una política gerencial que debe regir la actuación de una persona natural o jurídica, con el fin de construir y mantener una determinada imagen pública de tal persona, además de fomentar una actitud, intención o conducta favorable de sus públicos hacia ella. La política de las Relaciones Públicas debe estar sustentada en una investigación rigurosa y pertinente, y utilizar para conseguir sus fines diversas técnicas de comunicación persuasiva, que sin embargo pueden ser presentadas con otra apariencia.

En este caso, el término política, en lugar de recurrir a las expresiones filosofía o función, es preferible porque el vocablo político se ajusta perfectamente a la naturaleza y el carácter de las Relaciones Públicas. Una acepción de la palabra política incluye la

"habilidad o tacto en el manejo de los asuntos en que hay que tratar con gente" (Moliner, 1998),

al igual que incorpora las

"orientaciones o directrices que rigen la actuación de una persona o entidad en un asunto o campo determinado" (RAE, 2018).

Por otro lado, tanto los términos de "persona natural" como de "persona jurídica" implican un grupo de personas o una sola de ellas que pueden ser el referente y el emisor de las distintas acciones de Relaciones Públicas. Referir las Relaciones Públicas solamente a las personas jurídicas supondría un no deseado reduccionismo.

\section{Dimensión social de las Relaciones Públicas}

Con el paso del siglo XX al XXI se produjo una gran precisión en la definición de su marco conceptual y se adoptaron nuevos conceptos de tipo vanguardista. Una nueva línea epistemológica apareció en el horizonte de la investigación y la práctica de las Relaciones Públicas, entendidas casi por primera vez como una gestión empresarial socialmente responsable. No obstante, la nueva y original orientación de las Relaciones Públicas ya había sido apuntada años antes por algunos autores. Matrat (1971; 1975), en la órbita académica francófona, con una perspectiva humanista, antropológica y relacional, consideraba que las Relaciones Públicas debían ser entendidas como una política que en primer lugar debe conciliar el interés de la empresa y el interés de quienes ella depende; y sólo después, poner en marcha un plan de comunicación capaz de crear y mantener relaciones de confianza con todos los públicos de la empresa, tal y como recoge Xifra (2006). Sobre esta base descansa la llamada definición oficial de la "doctrina europea de las Relaciones Públicas" que subraya esencialmente su ineludible función social. Las Relaciones Públicas son, tanto de pensamiento como de acción, una política directiva de la empresa, o de cualquier otra estructura social, que tiene como objetivo crear un clima de confianza y afectos con sus públicos, siempre vinculado a la vigilancia constante de las opiniones existentes en su entorno. Para lograrlo la empresa se sirve de la difusión directa, o a través de los medios de comunicación, de informaciones honestas y completas sobre el parentesco entre las finalidades y los procedimientos de aquéllas y el bien común (Ugeux, 1976, p. 32-33; Xifra, 2006, p. 237).

En el panorama norteamericano, Harlow, de la Stanford University, promotor de las Relaciones Públicas científicas en Estados Unidos y fundador de la PRSA (Public Relations Society of America), tras un arduo y constante trabajo de investigación, en 1975 reunió a 65 líderes, académicos y profesionales, para analizar 472 definiciones sobre Relaciones Públicas, y a partir de sus deliberaciones, formuló su propia definición. 
“Las Relaciones Públicas son una función gerencial característica que ayuda a establecer y mantener líneas de mutua comunicación, entendimiento, aceptación y cooperación entre una organización y sus públicos; ayuda a mantener informada a la opinión pública y a despertar su interés; define y enfatiza la responsabilidad de la gerencia para servir al interés público; propicia una gerencia actualizada, al día, y efectivamente dispuesta al cambio, sirviendo como sistema de advertencia para anticipar tendencias y utiliza la comunicación basada en la investigación, el rigor y la ética como su principal herramienta" (Harlow, 1976, p. 36.).

Sin embargo, el carácter social de las Relaciones Públicas ya fue adelantado por Bernays (1990, p. 155) en los años veinte del siglo pasado al afirmar que son la práctica social de la responsabilidad:

“Cuando la profesión de las Relaciones Públicas fue perfilada, por vez primera, en mi libro titulado Crystallizing public opinion, fue imaginada tal como funcionaban otras profesiones: o sea, como un arte aplicado a una ciencia, en este caso a las ciencias sociales, y en el cual la motivación primordial era el interés público y no la motivación pecuniaria. Una empresa de Relaciones Públicas ética se rige por este principio" (Bernays, 1990, p. 155).

Otros conocidos estudiosos de las Relaciones Públicas, como Cutlip, Center y Broom ahondaron y profundizaron en este asunto con unos límites claros al afirmar que

"las Relaciones Públicas son una función gerencial que identifica, establece y mantiene relaciones mutuamente beneficiosas entre una organización y sus diferentes públicos" (Cutlip; Center; Broom, 1985, p. 4).

De esta forma, el concepto de gerencia responsable, con el ánimo de satisfacer no sólo los intereses persuasivos de la propia organización, sino también y fundamentalmente de servir a los intereses del público, se convirtió en el nuevo concepto de las Relaciones Públicas: las Ilamadas Relaciones Públicas excelentes, que responden al modelo más refinado y avanzado propuesto por Grunig (Grunig; Hunt, 1984; Grunig, 1992), bautizado con el nombre "bidireccional simétrico", basado en el entendimiento mutuo entre la organización y sus públicos. Bidireccional en su comunicación y simétrico por la búsqueda de resultados equilibrados que beneficien igualmente a la organización y a sus públicos. En este mismo sentido se pronunciaba el británico Jefkins cuando afirmaba que

"las Relaciones Públicas consisten en todas aquellas formas de comunicación planeadas, externas e internas, entre la organización y sus públicos con el propósito de alcanzar los objetivos específicos relativos al mutuo entendimiento" (Jefkins, 1995, p. 8).

Esta puntualización entendía las Relaciones Públicas excelentes como toda comunicación que tiene como objetivo conseguir el mayor y mejor entendimiento entre organizaciones y sus públicos.

Diversas instituciones internacionales han seguido esta misma perspectiva para dar cuerpo a la epistemología de las Relaciones Públicas. El British Institute of Public Opinion las definió como

"un esfuerzo intencionado, planificado y continuo para establecer y mantener el entendimiento mutuo entre una organización y sus públicos" (Wilcox; Ault; Agee, 1995, p. 6).

Por su parte, el Deutschen Public Relations Gesellschaft e.V. puso énfasis en los temas de la imprescindible fase de la investigación.

"Las Relaciones Públicas son el esfuerzo consciente y legítimo para lograr el entendimiento y establecer y mantener la confianza con el público sobre las bases de la investigación sistemática” (Wilcox; Ault; Agee, 1995, p. 6).

El Dansk PR-klub, de Dinamarca, siguió la misma trayectoria:

"Las Relaciones Públicas son el esfuerzo gerencial continuo y sistemático mediante el cual las organizaciones, privadas y públicas, pretenden establecer el entendimiento, la simpatía y el apoyo de aquellos públicos con los que tienen y esperan tener contacto" (Wilcox; Ault; Agee, 1995, p. 6).

Los términos esfuerzo gerencial, esfuerzo intencionado y esfuerzo consciente y legítimo prevalecen ante otras opciones, pero siempre destinados a conseguir la confianza, la colaboración y el mutuo entendimiento con los públicos posibles. Por su parte, Ehling (1992) dejó muy claro que sólo se puede hablar de su función gerencial si actúan como tal, en clara diferencia con otras actividades organizacionales como la mercadotecnia, la gerencia de personal o la administración. Asimismo, añadió que solamente se puede conseguir este estatus cumpliendo su más importante misión: mantener el mutuo acuerdo entre la organización, sus públicos y otras organizaciones, además de hacer crecer el entorno social donde se hallan inmersos públicos y organizaciones.

De igual manera, Noguero-Grau, recogiendo la definición propuesta por la International Public Relations Association (IPRA), afirmó que las

“Relaciones Públicas son una actividad de dirección de carácter permanente y organizado, por la cual la empresa u organismo privado o público pretende conseguir y mantener la comprensión, la simpatía y el concurso de aquellos con los que tiene o puede tener relación; en este punto, deberá adaptar en lo posible su conducta y, a través de una extensa información, obtener una comprensión más eficaz que tenga en cuenta los intereses comunes" (Noguero-Grau, 1995, p. 60). 


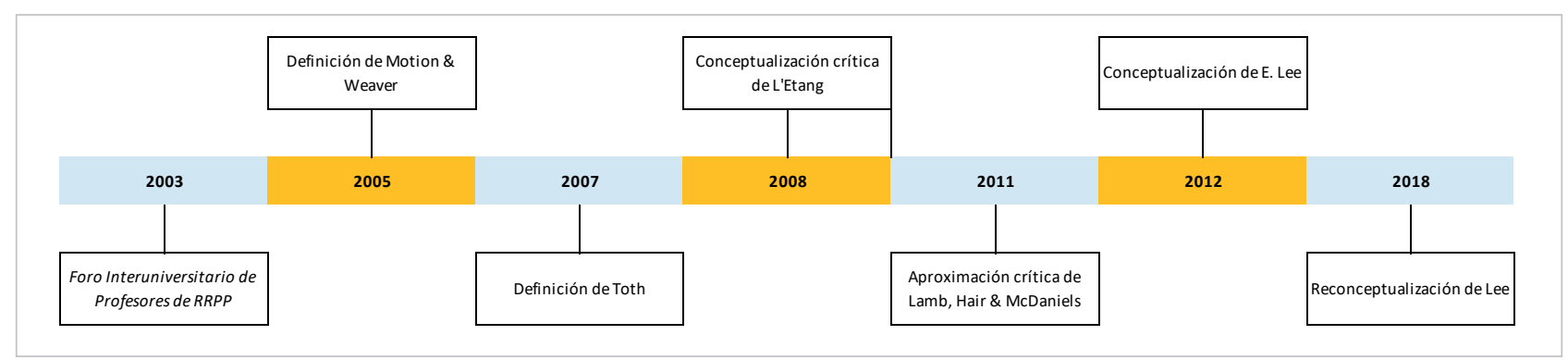

Cronograma 2. Evolución de la conceptualización de las RRPP en el siglo XXI.

Por otro lado, García-Nieto definió las Relaciones Públicas de vanguardia como

"una política gerencial socialmente responsable que debe regir la actuación de una persona natural o jurídica, con el fin de alcanzar y mantener el mutuo entendimiento entre ella y sus públicos" (García-Nieto, 1999, p. 35).

En esta misma línea se han mantenido la mayoría de las definiciones formuladas en los últimos años tanto en el panorama nacional como en el internacional. Heath afirma que

“las Relaciones Públicas son una función gerencial que planifica, investiga, comunica, promueve y colabora en la toma de decisiones para que la organización sea capaz de escuchar, valorar y dar respuesta apropiada a las personas y grupos, con quienes la organización ha de mantener relaciones mutuamente beneficiosas para apoyar y favorecer el logro de su misión" (Heath, 2007, p. 43).

Por su parte, el Primer Foro Interuniversitario de Profesores de Relaciones Públicas (FIP-RRPP), que se reunió en la ciudad de Vic en 2003, las define como

"una disciplina científica que estudia la gestión del sistema de comunicación a través del cual se establecen relaciones de adaptación e integración entre una organización o persona y sus públicos (FIP-RRPP, 2003).

La PRSA, tras dos fracasados intentos en 2003 y 2007, con la colaboración de una docena de instituciones del sector, emprendió en 2012 la campaña Public relations defined para formular una nueva definición de Relaciones Públicas, que actualizara su enunciación vigente desde 1982:

"Las Relaciones Públicas ayudan a una organización y sus públicos a adaptarse mutuamente los unos a los otros".

Así, entre el 13 y el 26 de febrero de 2012, se llevó a cabo una votación pública en la que participaron 1.447 expertos, de la que resultó ganadora con el $46,8 \%$ de los votos la definición que estimaba que

"las Relaciones Públicas son un proceso estratégico de comunicación que construye relaciones mutuamente beneficiosas entre las organizaciones y sus públicos".

No obstante, esta nueva definición ha sido objeto de gran controversia de modo que, a ojos de no pocos expertos, se perfila más acertada alguna de las otras dos formulaciones finalistas:

"Las Relaciones Públicas son una función de dirección consistente en investigar, comunicar y colaborar con los públicos para construir relaciones mutuamente beneficiosas"

y

"Las Relaciones Públicas consisten en el proceso estratégico de interacción entre las organizaciones y sus públicos para lograr el entendimiento mutuo y alcanzar objetivos".

Reducir las Relaciones Públicas a un proceso estratégico de comunicación significa obviar su función directiva y gerencial, lo cual supone, sin duda alguna, una vuelta atrás en la concepción científica de las Relaciones Públicas. Podría afirmarse que el elemento de anclaje a la actualidad de la nueva definición propuesta por la PRSA es la búsqueda de objetivos simétricos y equilibrados al pretender relaciones mutuamente beneficiosas para la organización y sus públicos. Sin embargo, renunciar al carácter gerencial de las Relaciones Públicas implica aceptar su retroceso conceptual, y la cesión de su posición en la estructura y en el organigrama de las organizaciones.

\section{Las Relaciones Públicas no son marketing}

Cabe preguntarse si este retroceso es casual o es el resultado de la influencia de la participación de algunas asociaciones del mundo del marketing que colaboraron con la PRSA en la definición de Relaciones Públicas. Tradicionalmente, los estudiosos de marketing han considerado a las Relaciones Públicas como una más de las herramientas de su mezcla promocional, junto con la publicidad, la promoción de ventas o la venta personal (Stanton; Etzel; Walker, 2007, p. 506), y el marketing como vía para "satisfacer necesidades de forma rentable" (Kotler; Keller, 2006, p. 6).

Lamb, Hair y McDaniel (2011, p. 534) estiman que las Relaciones Públicas son la función de marketing que evalúa las actitudes públicas, identifica temas que pueden producir una preocupación pública y ejecuta programas de acción para 
lograr la comprensión y aceptación públicas. Las Relaciones Públicas son la función de marketing que:

- investiga las actitudes de los públicos;

- identifica las áreas dentro de la organización en las que el público puede interesarse;

- emprende un programa de acción para lograr su comprensión y su aceptación.

Las Relaciones Públicas ayudan a una organización a comunicarse con sus clientes, proveedores, accionistas, funcionarios del gobierno, empleados y con la comunidad en donde opera. Las empresas las utilizan no sólo para mantener una imagen positiva, sino también para educar al público acerca de las metas y los objetivos de la empresa, introducir nuevos productos y ayudar a apoyar el esfuerzo de ventas. Según Lamb, Hair y McDaniel (2011, p. 534), las Relaciones Públicas constituyen igualmente una pieza más del entramado de la llamada comunicación integral de marketing (CIM).

"La comunicación integral de marketing es la coordinación cuidadosa de todos los mensajes promocionales, publicidad tradicional, marketing directo, interactivo, Relaciones Públicas, promoción de ventas, venta personal, marketing de eventos y otras comunicaciones, para un producto o servicio, con el fin de asegurar la consistencia de los mensajes en cada punto de contacto en donde una empresa se encuentra con el consumidor" (Lamb; Hair; McDaniel, 2011, p. 538).

Pues bien, aún en el caso de las definiciones del marketing que enfatizan el aspecto de interrelación entre la empresa y sus clientes, como las que citamos a continuación, debemos dejar claro que las Relaciones Públicas de ninguna manera pueden considerarse una función, instrumento, o parte del marketing, de la misma forma que el marketing no forma parte de las Relaciones Públicas. Aunque los profesionales de las Relaciones Públicas asesoren y colaboren en la planificación, ejecución y evaluación de campañas o acciones de carácter comercial, en modo alguno las Relaciones Públicas pueden considerarse una pieza más de la mercadotecnia.

La American Marketing Association revisó su definición de marketing en 2004.

"Marketing es una función organizacional y un conjunto de procesos de creación, comunicación y entrega de valor al cliente y de administración de relaciones con los clientes de manera que se beneficie a la organización y a sus grupos de interés" (Keefe, 2004, p. 17).

El marketing es el proceso mediante el cual las empresas crean valor para sus clientes y generan relaciones con ellos para recíprocamente captar el valor de sus clientes. Por otro lado,

"la administración de las relaciones con los clientes es el proceso general de crear y mantener relaciones rentables con los clientes al entregar satisfacción y valor superior al cliente. Maneja todos los aspectos de adquirir, mantener y crecer a los clientes" (Armstrong; Kotler, 2013, p. 13).

La incursión mercadotécnica, como se comprueba, lleva a la ambigüedad, a la instrumentalización y al reduccionismo de las Relaciones Públicas, de su naturaleza, su función y sus objetivos, dotándolas de un carácter comercial y económico del que por principio las Relaciones Públicas deben permanecer ajenas. En la mayoría de las empresas las Relaciones Públicas no son responsabilidad del departamento de marketing. Si hay un esfuerzo organizado, por lo regular lo maneja un pequeño departamento de Relaciones Públicas que reporta directamente a la dirección general (Stanton; Etzel; Walker, 2007, p. 574).

\section{Conclusiones. El desafío conceptual de las Relaciones Públicas}

Una primera conclusión general es que, como se ha podido constatar, la mayoría de las definiciones de Relaciones Públicas responden al enfoque funcionalista del paradigma dominante de las Relaciones Públicas, fundamentado en las aportaciones del estudio Excellence in public relations and communication management dirigido por Grunig (1992), fundador de la llamada Escuela de Maryland. Sin embargo, incluso considerando las argumentaciones de los representantes de la corriente crítica de la teoría de las Relaciones Públicas, L’Etang (2008), Edwards (2012; 2018) o Motion y Weaver (2005), que postulan una aproximación sociocultural a las Relaciones Públicas, y la necesidad de analizar la importancia creciente de esta disciplina en su contexto social, cultural, político y económico, estas definiciones mantienen su validez epistemológica.

Tras la revisión conceptual y diacrónica, se comprueba que en la década de 1970, pese a la falta de acuerdo en la definición de Relaciones Públicas, existe ya un común reconocimiento de su naturaleza persuasiva y de su fundamentación en las ciencias sociales. En la siguiente década, las Relaciones Públicas, influenciadas por las teorías de la dirección empresarial, se identifican como una función gerencial y directiva de la comunicación. Y
Este trabajo confirma la naturaleza gerencial de las Relaciones Públicas, sus objetivos de mutuo beneficio y recíproco entendimiento entre la organización y sus públicos, y su carácter socialmente responsable 
a partir de los años noventa, avanzan socialmente hacia unos objetivos simétricos de mutuo entendimiento con los públicos. Estas últimas definiciones, que reafirman el carácter gerencial, coexisten a su vez con otras que las conciben como un proceso estratégico de comunicación.

Este trabajo confirma la naturaleza gerencial de las Relaciones Públicas, sus objetivos de mutuo beneficio y recíproco entendimiento entre la organización y sus públicos, y su carácter socialmente responsable. Las Relaciones Públicas son ajenas a la naturaleza y a los objetivos comerciales y económicos del marketing, con el que a veces equivocadamente se confunden. Ni las Relaciones Públicas forman parte del marketing, ni el marketing forma parte de las Relaciones Públicas.

En síntesis, pueden formularse las siguientes conclusiones:

1. Las Relaciones Públicas constituyen una política gerencial que implica la planificación estratégica para la consecución de unos resultados, fundamentada en la investigación científica, traducida en decisiones y acciones, y proyectada con planteamientos coherentes de acuerdo con unos parámetros temporales y presupuestarios.

2. La mayor parte de las definiciones formuladas reconocen indudablemente la función directiva de las Relaciones Públicas. El plan de Relaciones Públicas compromete el comportamiento de la organización y de las personas que la forman, por eso el departamento de Relaciones Públicas, localizado como órgano staff, debe asesorar directamente a la presidencia y a la alta dirección de la organización.

3. Los principales objetivos de las Relaciones Públicas consisten en crear, establecer, obtener, suscitar, mantener y mejorar el entendimiento, la confianza y la comprensión recíproca con los públicos, fundamentados en el beneficio mutuo de la organización y los diferentes grupos sociales con los que se relaciona.

4. Los objetivos persuasivos de las Relaciones Públicas, derivados de los principales, se plasman en la construcción de la opinión pública, en general, y la opinión de los públicos internos y externos, en particular.. Estos objetivos se concretan en la creación, modificación y refuerzo de la notoriedad, imagen, actitud, aceptación, intención, conducta y hábito de conducta de los públicos hacia la organización.

5. Las Relaciones Públicas científicas y socialmente responsables se fundamentan en la planificación de la comunicación, en sus diligencias negociadoras y mediadoras y en sus aplicaciones persuasivas. La mediación, la negociación y la comunicación persuasiva son los principales instrumentos de las Relaciones Públicas para la consecución de sus objetivos, erigiéndose como sus elementos proveedores de sentido.

6. Los sujetos de las Relaciones Públicas pueden ser personas naturales o jurídicas: empresas, organizaciones, grupos, entidades, tanto de índole público como privado y personas físicas.

7. Los públicos externos receptores y objetivo de las Relaciones Públicas se amplían inexorablemente al concebirlas como política gerencial socialmente responsable. Los límites se dilatan al incluir en su consideración como públicos objetivo a las personas beneficiarias de las acciones del ejercicio de la responsabilidad social de la organización. Personas que de otro modo nunca se relacionarían con la empresa, al no mantener con ella vínculos o lazos de ningún otro tipo, productivo, económico, o ni siquiera persuasivo.

8. Las Relaciones Públicas presentan la naturaleza indiscutible de cooperar y colaborar en la vida social de una forma beneficiosa, con la mejora recíproca de los actores en su proceso gerencial y comunicacional.

Para terminar esta etapa del estatuto epistemológico de las Relaciones Públicas entendidas como Relaciones Públicas socialmente responsables, cabe subrayar algunos puntos clave:

- Las Relaciones Públicas son una política socialmente responsable que debe regir la actuación de una persona natural o jurídica, con el fin de alcanzar y mantener el mutuo entendimiento entre una organización y sus públicos.

- Esta política gerencial se establece con el fin de responder prioritariamente a los intereses del público, sin renunciar al objetivo de formar, mantener y mejorar una determinada imagen pública de dicha persona, así como una actitud, intención o conducta favorable hacia ella de sus públicos, basándose en la investigación científica pertinente.

- Para conseguir estos fines se pueden utilizar los métodos propios de la mediación y la negociación, así como todo tipo de técnicas, comunicativas persuasivas -aunque éstas se puedan presentar con cualquier otra apariencia- y no comunicativas, contando entre ellas las acciones específicas del ejercicio de la responsabilidad social corporativa.

\section{Referencias}

Armstrong, Gary; Kotler, Philip (2013). Fundamentos de marketing. 11 ed. Naucalpan de Juárez (México): Prentice Hall. Pearson Education. ISBN: 9786073217224

Asamblea Mundial de Asociaciones de Relaciones Públicas (1978). Declaración de México. Primera Asamblea Mundial de Asociaciones de Relaciones Públicas. México, agosto. 
Bernays, Edward (1923). Crystallizing public opinion. New York: Boni \& Liveright. ISBN: 9781935439264

Bernays, Edward (1970). Public relations. 5 th ed. Oklahoma: University of Oklahoma Press. ISBN: 9780806114576

Bernays, Edward (1990). Los años últimos: radiografía de las Relaciones Públicas (1956-1986). Barcelona: ESRP-PPU. ISBN: 9788476656587

Bettinghaus, Erwin (1987). Persuasive communication, $4^{\text {th }}$ ed. New York, Holt: Rinehart and Winston. ISBN: 9780 030665707

Carlson, Robert (1968). "Public relations". In: International encyclopaedia of social sciences. London: The Macmillan \& Press, pp 208-2017. [Versión castellana: "Relaciones Públicas". En: Enciclopedia internacional de las ciencias sociales. Madrid: Aguilar, 1979, 2a reimp. ISBN: 97884 03189966]

Center, Allen; Jackson, Patrick (1995). Public relations practices. Managerial, case studies and problems. Englewood Cliffs: N. J.: Prentice-Hall. ISBN: 9780133127645

Cutlip, Scott; Center, Allen (1978). Effective public relations, $5^{\text {th }}$ ed. Englewood Cliffs: N.J.: Prentice-Hall. ISBN: 978 0132450355

Cutlip, Scott; Center, Allen; Broom, Glen (1985). Effective public relations. Revised $6^{\text {th }}$ ed. Englewood Cliffs, N. J.: Prentice-Hall. ISBN: 9780132450775

Edwards, Lee (2012). "Defining the 'object' of public relations research: A new starting point". Public relations inquiry, v. 1, n. 1, pp. 7-30. https://doi.org/10.1177/2046147X11422149

Edwards, Lee (2018). Understanding public relations: Theory, culture and society. London: SAGE Publications Ltd. ISBN: 9781473913103

Ehling, William (1992). "Education and professionalism". In: Grunig, James E. (ed.). Excellence in Public Relations and communication management. N. Y.: Hillsdale, Lawrence Erlbaum Associates, pp. 439-464. ISBN: 9780805802276

Foro Interuniversitario de Profesores de RRPP (2003). Reunión del I Foro Interuniversitario de Profesores de RRPP. Universitat de Vic. Barcelona.

García-Nieto, María-Teresa (1999). "La propaganda como fuente de las Relaciones Públicas". Historia y comunicación social, v. 4, pp. 35-46.

https://revistas.ucm.es/index.php/HICS/article/view/HICS9999110035A

García-Orosa, Berta (2019). "25 years of research in online organizational communication. Review article". El profesional de la información, v. 28, n. 5, e280517.

https://doi.org/10.3145/epi.2019.sep.17

Grunig, James E. (ed.) (1992). Excellence in public relations and communication management. N.Y.: Hillsdale, Lawrence Erlbaum Associates. ISBN: 9780805802276

Grunig, James E.; Hunt, Todd (1984). Managing public relations. N.Y.: Holt Rinehart \& Winston. ISBN: 9780030583377

Harlow, Rex F. (1976). "Building a public relations definition". Public relations review, v. 2, n. 4, pp. 34-42. https://doi.org/10.1016/S0363-8111(76)80022-7

Heath, Robert-Lawrence (2007). "Management through advocacy: Reflection rather than domination". In: Toth, Elizabeth L. (ed.). The future of excellence in Public Relations and communication management. Challenges for the next generation. New Jersey: Lawrence Erlbaum Associates, pp. 41-66. ISBN: 9780805855951

Hellín-Ortuño, Pedro; Trindade, Eneus; García-López, Javier (2019). "Latin-American perceptions on definitions and arguments about crossmedia and transmedia in advertising". El profesional de la información, v. 28, n. 4, e280408.

https://doi.org/10.3145/epi.2019.jul.08

Jefkins, Frank (1995). Public relations, $4^{\text {th }}$ ed., $3^{\text {th }}$ reprint. London: Pitman Publishing. ISBN: 9780712117098

Keefe, Lisa M. (2004). "What is the meaning of 'marketing'?”. Marketing news, 15 September, p. 17.

Kotler, Philip; Keller, Kevin-Lane (2006). Marketing management, $12^{\text {th }}$ ed. New Delhi: Prentice Hall. ISBN: 9780 131457577

Lamb, Charles; Hair, Joseph; McDaniel, Carl (2011). Marketing. Santa Fe (México): Cengage Learning Editores. ISBN: 978 1439039427

Lesly, Philip (1981). "Report and recommendations: Task force on structure and role of public relations". Public relations journal, March, p. 32. 
L’Etang, Jacquie (2008). Relaciones Públicas. Conceptos, práctica y crítica. Barcelona: UOC Press. ISBN: 9788497888240

Manfredi-Sánchez, Juan-Luis (2020). "Globalization and power: the consolidation of international communication as a discipline. Review article". El profesional de la información, v. 29, n. 1, e290111.

https://doi.org/10.3145/epi.2020.ene.11

Mañas-Viniegra, Luis; Jiménez-Gómez, Isidro (2019). “Evolución del perfil profesional del community manager durante la década 2009-2018”. El profesional de la información, v. 28, n. 4, e280403.

https://doi.org/10.3145/epi.2019.jul.03

Marston, John (1979). Modern public relations. New York: McGraw-Hill. ISBN: 9780070406193

Matilla, Kathy; Cuenca-Fontbona, Joan; Compte-Pujol, Marc (2017). "An analysis of the university curricula of Spanish 'dircoms' from a public relations approach". Public relations review, v. 43, n. 3, pp. 624-634.

https://doi.org/10.1016/j.pubrev.2017.04.003

Matrat, Lucien (1971). Relations publiques et management, Bruselas, CERP.

Matrat, Lucien (1975). "Doctrine européenne des relations publiques, condition du dialogue et de la participation". Estudios de comunicación social y relaciones públicas, v. 1, pp. 29-33.

Moliner, María (1998). Diccionario de uso del español, 2ạ ed. Madrid: Gredos ISBN: 9788424913441

Moore, Frazier; Canfield, Bertrand (1980). Relaciones públicas. Principios, casos y problemas. México: Compañía Editorial Continental. ISBN: 9789682602030

Motion, Judy; Weaver, C. Kay (2005). “A discourse perspective for critical public relations research: Life sciences network and the battle for truth". Journal of public relations research, v. 17, n. 1, pp. 49-67.

https://doi.org/10.1207/s1532754xjprr1701_5

Noguero-Grau, Antoni (1995). La función social de las Relaciones Públicas: historia, teoría y marco legal. Barcelona: ESRP-EUB. ISBN: 9788447705153

RAE (2018). "Política". Diccionario de la lengua española. Real Academia Española.

https://dle.rae.es/?id=Ta2HMYR

Stanton, William J.; Etzel, Michael J.; Walker, Bruce J. (2007). Fundamentos de marketing. 14 ed. Mcgraw-Hill/Interamericana Editores. ISBN: 9789701062019

Ugeux, William (1976). Las Relaciones Públicas: una nueva función social. Barcelona: Seix Barral. ISBN: 844002049 X

Westphalen, Marie-Hélène; Piñuel-Raigada, José-Luis (eds.) (1993). La dirección de comunicación: Prácticas profesionales. Diccionario técnico. Madrid: Ediciones del Prado. ISBN: 8478383832

Wilcox, Dennis L.; Ault, Philip H.; Agee, Warren K. (1995). Public relations strategies and tactics. $4^{\text {th }}$ ed. New York: Harper Collins. ISBN: 9780673993090

Xifra, Jordi (2006). "Lucien Matrat y la consolidación de las Relaciones Públicas en Europa”. Historia y comunicación social, v. 11 , p. 229-240.

https://revistas.ucm.es/index.php/HICS/article/view/HICS0606110229A

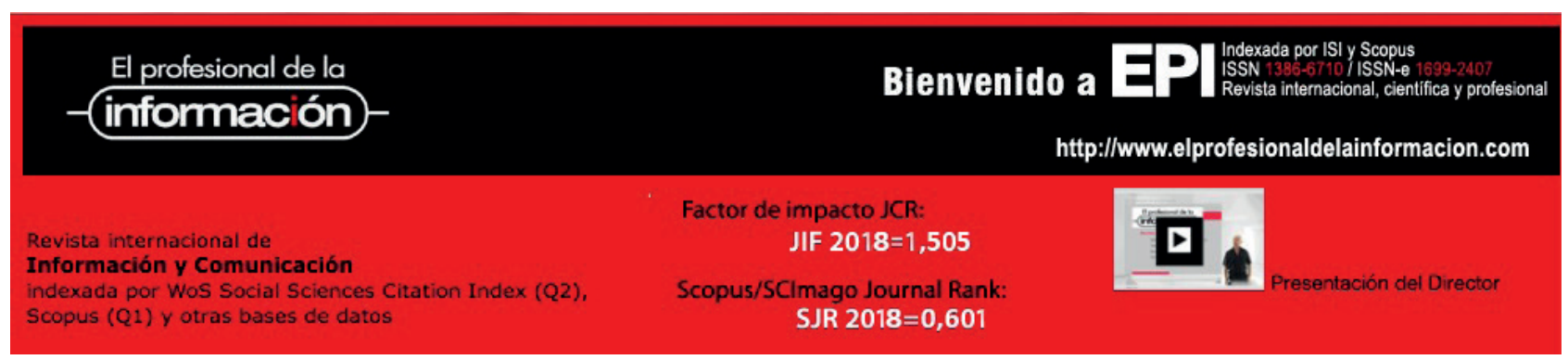

